\title{
BMP4 knockdown of NCSCs leads to aganglionosis in the middle embryonic stage
}

\author{
HONG-BO LI，XIAN-QING JIN，XIN JIN，ZHENG-HUA GUO，XIONG-HUI DING， \\ QUAN WANG and RUI-ZHUO LIU
}

\begin{abstract}
Department of Pediatric Surgery, Children's Hospital of Chongqing Medical University, Ministry of Education Key Laboratory of Child Development and Disorders, China International Science and Technology Cooperation Base of Child Development and Critical Disorders, Chongqing Key Laboratory of Pediatrics, Chongqing 400014, P.R. China
\end{abstract}

Received September 23, 2017; Accepted December 14, 2017

DOI: $10.3892 / \mathrm{mmr} .2018 .8519$

\begin{abstract}
Transplacental bone morphogenetic protein (BMP)4 RNA interference (RNAi) is a technique used to knockdown genes in embryos. BMP4 are essential for the development of nervous system in the differentiation of neural crest stem cells (NCSCs). The failure of differentiation and migration of NCSCs may lead to aganglionosis. In the present study, pregnant mice were divided into three groups: Ringer's group, pSES group and RNAi-BMP4 group. In order to silence the BMP4 gene in the first generation (F1), 11.5 day pregnant mice were injected with the small interfering RNA BMP4 plasmid, pSES or Ringer's solution via the tail vein. Semi-quantitative reverse transcriptase-polymerase chain reaction (RT-PCR)and western blotting were employed to ensure the downregulation of BMP4. Finally, $\mathrm{X}$-rays were performed following a barium enema. Aganglionosis was diagnosed by general anatomy and immunohistochemistry. Compared with the control group, transplacental RNAi was able to downregulate the BMP4-Smad4 of 11.5 day embryos, as determined by semi-quantitative RT-PCR and western blotting. The megacolons of the mice were demonstrated by X-ray and confirmed by general anatomy. Aganglionosis of colonic mucosa and submucosa were diagnosed by pathology, and immunohistochemistry. Knockdown of BMP4 in pregnant mice at the middle embryonic stage led to aganglionosis. It was therefore demonstrated that BMP-Smad was essential to the NCSCs of middle stage embryos. BMP-Smad served important roles in the generation of aganglionosis. This technique of knockdown
\end{abstract}

Correspondence to: Dr Xin Jin, Department of Pediatric Surgery, Children's Hospital of Chongqing Medical University, Ministry of Education Key Laboratory of Child Development and Disorders, China International Science and Technology Cooperation Base of Child Development and Critical Disorders, Chongqing Key Laboratory of Pediatrics, 136 2nd Road, Yuzhong, Chongqing 400014, P.R. China

E-mail: jinxincq@163.com

Key words: knockdown mouse model, aganglionosis, neural crest stem cells
BMP4 gene may be used to establish an aganglionosis mouse model.

\section{Introduction}

Aganglionosis or Hirschsprung's disease (HD) is a congenial intestinal dynamic disorder characterized by intestinal submucosal and myenteric plexus parasympathetic ganglion cell loss, leading to persistent diseased colonic convulsions, and contractions in addition to the obstruction of intestinal contents (1-4). This disease is a colonic motor disorder with an incidence of 1:5,000 live births worldwide (5-7). In previous studies, the failure of neural crest stem cells (NCSCs) todifferentiate and migrate was demonstrated to be associated with the poor development of the enteric nervous system, and identified as the main cause of aganglionosis $(8,9)$.

Bone morphogenetic proteins (BMPs) are among the most important proteins involved in the development of the enteric nervous system (8-12). BMP/Smad signalling serve a key role in NCSC migration at an early embryonic stage, while NCSC differentiation occurs during the middle embryonic stages $(8,11)$. Eventually, BMP/Smad signalling determines the function of the intestinal nervous system (10-12). The current studies were designed to investigate the effects of the downregulation of $B M P 4$ gene expression in NCSCs using a transgenic mouse model in which low expression level of the BMP4 gene was governed by RNAi-BMP genomic sequences (13-16). A series of pregnant mice at 6.5-14.5 days post coitum (dpc) with post-implantation staged mouse embryos were injected via the tail vein with the pSES-small interfering RNA (si)BMP4 plasmid to silence the $B M P 4$ gene, and these procedures were applied to establish an animal model (14). One of the objectives of the present study was to expand current knowledge on the effect of BMP4 on NCSCs during the middle stage of embryo development. The current study also aimed to assess the neurodevelopmental abnormalities associated with the knockdown of BMP4.

\section{Materials and methods}

Animals and experimental groups. Balb/c mice (male 12, female 36; 8-12 weeks old; weight 14-18 g) were purchased 
from the Animal Center of Chongqing Medical University (Chongqing, China). The mice were kept in a specific pathogen-free facility room at the Chongqing Children's Hospital Animal Center (Chongqing, China), with 50 $\pm 5 \%$ humidity and a temperature of $25 \pm 2^{\circ} \mathrm{C}$. Food and water were provided ad libitum. Male and female mice were kept in single cages at a 1:2-1:4 ratio. A female mouse in which a vaginal plug was identified the next dawn was marked as $0.5 \mathrm{dpc}$ and housed separately. The present study was approved by The Ethics Committee of Chongqing Medical University.

Tail vein injections. Pregnant mice were randomly divided into the following groups: Ringer's group $(n=12)$, pSES group $(n=12)$ and RNAi-BMP4 group $(n=12)$; these were injected with $10 \mu \mathrm{l} / \mathrm{g}$ Ringer's solution, $50 \mathrm{ng} / \mu \mathrm{l} \mathrm{pSES}$ empty vector and $50 \mathrm{ng} / \mu \mathrm{l} \mathrm{pSES-SiBMP4}$ vector, respectively. The pSES vectors bore a copy of the entire DsRed coding region, allowing fluorescent detection of the delivered plasmids. At $11.5 \mathrm{dpc}$, the solution (at a volume of $10 \mu \mathrm{l} / \mathrm{g}$ ) was injected into the tail vein. A $31 \mathrm{G}$ needle was used and the injectionusually performed within $5 \pm 1 \mathrm{sec}$. Plasmids for siRNA were purchased from the Oncogene Laboratory, Biological Sciences Division, University of Chicago and contained the following 4 sites of RNAi to silence the BMP4 gene, lowercase is the gene match to the cutting site): (5'aGGTCCAGGAAGAAGAATAAt ttt3', mouse BMP4 simBMP4-site 1, sense strand; 3'aTTATT CTTCTTCCTGGACCttt5', mouse BMP4 simBMP4-site 1, antisense strand. 5'aGAGCCATGCTAGTTTGATAtttt3', mouse BMP4 simBMP4-site 2, sense strand; 3'aTATCAAAC TAGCATGGCTCtttt5', mouse BMP4 $\operatorname{sim} B M P 4$-site 2, antisense strand. 5'aGGGAAAAGCAACCCAATTAtttt3', mouse BMP4 simBMP4-site 3, sense strand; 3'aTAATTGGGTTG CTTTTCCCtttt5', mouse BMP4 simBMP4-site 3, antisense strand. 5'aGGGAAAAGCAACCCAATTAtttt3', mouse BMP4 simBMP4-site 4, sense strand; 3'aTAATTGGGTTGCTTTTC CCtttt5', mouse BMP4 simBMP4-site 4, antisense strand). At 1week [Ringer's group F1 mice $(n=16)$, pSES group $(n=18)$ and RNAi-BMP4 group $(\mathrm{n}=10)], 2$ weeks [Ringer's group $(n=15)$, pSES group $(n=15)$ and RNAi-BMP4 group $(n=12)]$ and 4 weeks [Ringer's group $(n=21), p S E S$ group $(n=15)$ and RNAi-BMP4 group $(n=7)]$ following birth, the F1 mice were sacrificed by cervical dislocation. Then, the target tissues were removed and rinsed in PBS at $4^{\circ} \mathrm{C}$. Parts of the tissues were stored at $-80^{\circ} \mathrm{C}$ and used for western blotting. The remaining samples were stored at $4-20^{\circ} \mathrm{C}$ in $4 \%$ paraformaldehyde.

Reverse transcriptase-semi-quantitative polymerase chain reaction ( $R T$-sqPCR) detection of BMP4 and Smad4 genes. Total RNA was extracted from the colon using TRIzol reagent (Invitrogen; Thermo FisherScientific,Inc., Waltham,MA,USA) according to the manufacturer's protocol. To generate cDNA, RT was performed with Prime Script RT Enzyme mix reverse transcriptase at $4^{\circ} \mathrm{C}$ (Invitrogen; Thermo Fisher Scientific, Inc.). Then, the cDNA samples were amplified by PCR using the following cycling conditions: $94^{\circ} \mathrm{C}$ for $5 \mathrm{~min}$; followed by 39 cycles at $94^{\circ} \mathrm{C}$ for $30 \mathrm{sec} ; 58^{\circ} \mathrm{C}$ for $30 \mathrm{sec} ; 72^{\circ} \mathrm{C}$ for $30 \mathrm{sec}$; and a final step at $72^{\circ} \mathrm{C}$ for $5 \mathrm{~min}$. Oligonucleotide primers were purchased from Invitrogen (Thermo Fisher Scientific, Inc.) as follows: BMP4 forward (F), GACTTCGAGGCG ACACTTCT and reverse (R), CCTGGGATGTTCTCCAGA
TG; Smad4 F, CATTCCAGCCTCCCATTTCCAATC and R, CACATAGCCATCCACAGTCACAAC; $\beta$-actin F, AAG ATGACCCAGATCATGTTTGAGACC and R, GCCAGG TCCAGACGCAGGAT. The amplified products were resolved in ethidium bromide-stained $2 \%$ agarose gels. Densitometry was performed using Quantity One software version 4.6.2. (Bio-Rad Laboratories, Inc., Hercules, CA, USA).

Western blotting analysis of BMP4. Protein extracts were prepared from the colon. Tissue samples were homogenized in RIPA lysis buffer and phenylmethanesulfonyl fluoride (Beyotime Institute of Biotechnology, Shanghai, China), and proteins were directly extracted according to the manufacturer's protocols. Protein concentrations were determined using a Micro Bicinchoninic Acid Protein Assay reagent (Beyotime Institute of Biotechnology). Protein samples were then diluted to obtain equal $(50 \mu \mathrm{g})$ protein amounts and heated at $100^{\circ} \mathrm{C}$ in an equal volume of SDS loading buffer (Beyotime Institute of Biotechnology) for $10 \mathrm{~min}$. Proteins were then separated by SDS-PAGE (5\% spacer gel, $40 \mathrm{~V}, 50 \mathrm{~min}$; $8 \%$ separating gel, $80 \mathrm{~V}, 70 \mathrm{~min})$. Proteins were then electrophoretically transferred (Bio-Rad Laboratories, Inc.) onto polyvinylidene fluoride membranes (EMD Millipore, Billerica, MA, USA) for $1.5 \mathrm{~h}$ at $250 \mathrm{~mA}$. To block non-specific binding, the membranes were incubated with 5\% bovine serum albumin (Kang Yuan Biology, Tianjing, China) in Tris-buffered saline with Tween $20(250 \mu \mathrm{l}$ Tween 20 in $500 \mathrm{ml} \mathrm{PBS}$ ) at $37^{\circ} \mathrm{C}$ for $1.5 \mathrm{~h}$. The membranes were then incubated overnight at $4^{\circ} \mathrm{C}$ with rabbit anti-BMP4 primary polyclonal antibody (GR49989-1; 1:400; Abcam, Cambridge, MA, USA) and $\beta$-actin (4AH240911; 1:150; 4A Biotech Co., Ltd., Beijing, China.). Next, the membranes were incubated at $4^{\circ} \mathrm{C}$ for $1 \mathrm{~h}$ with peroxidase-conjugated secondary anti-rabbit IgG (TA130015; 1:4,000; OriGene Technologies, Inc., Beijing, China), according to the manufacturer's protocol. The protein of interest was visualized using an enhanced chemiluminescence western blotting substrate (Boster Biological Technology, Pleasanton, CA, USA) and its relative expression was quantified using a Chemidoc XRS gel imaging system Quantity One software version 4.6.2. (Bio-Rad Laboratories, Inc.).

Immunohistochemistry. Colontissue was immediately fixed in $4 \%$ buffered formalin for $48 \mathrm{~h}$, embedded in paraffin, and sectioned at $5 \mu \mathrm{m}$. Antigen retrieval was performed by boiling the sections in $0.01 \mathrm{M}$ sodium citrate in $1 \mathrm{~L} \mathrm{PBS}(\mathrm{pH} 7.4)$ followed by a $20 \mathrm{~min}$ incubation at room temperature, in $3 \%$ $\mathrm{H}_{2} \mathrm{O}_{2}$ for 20 min and blocked in 5\% BSA (Kangyuan Biology, Tianjing, China) for $20 \mathrm{~min}$ at room temperature. Following incubation in $5 \%$ normal serum for $20 \mathrm{~min}$ at room temperature, sections were incubated with rabbit anti-BMP4 primary polyclonal antibody overnight at $4^{\circ} \mathrm{C}$ (GR49989-1, ab39973; 1:500; Abcam). Slides were then stained with goat anti-rabbit secondary antibody (PV-6001, 1:1,500 dilution) from OriGene Technologies, Inc. (Beijing, China) for $1 \mathrm{~h}$ at room temperature. Detection was accomplished using a DAB kit (Beyotime Institute of Biotechnology, Shanghai, China). Positive staining was assessed by the degree of brown colour development. The integrated optical density of positive staining was measured by NIS-Elements Viewer version 4.0 (Nikon Corporation, Tokyo, Japan) using an Eclipse 55i microscope (x40; Nikon Corporation). 

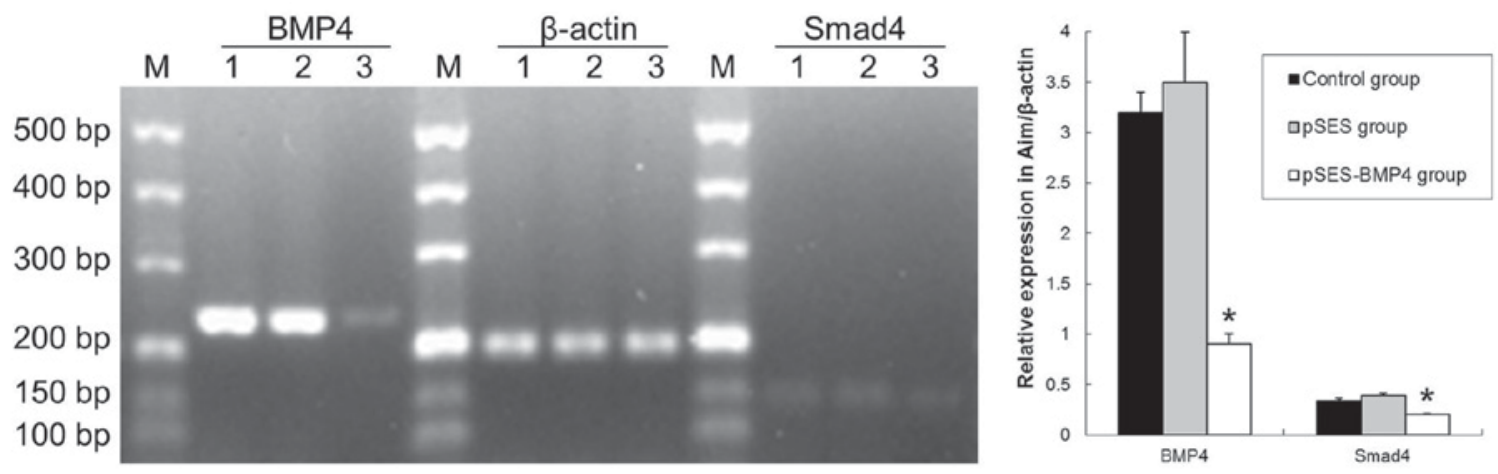

Figure 1. Semi-quantitative reverse transcriptase-polymerase chain reaction of the different groups of Balb/C mouse colons. Lane 1: Ringer's group, Lane 2: pSES group, Lane 3, RNAi-BMP4 group and M, marker. At the RNA level, the RNAi-BMP4-induced mice, in contrast with the control group, significantly downregulated the levels of BMP4 and Smad4 in the colon. "P<0.05. RNAi, RNA interference; BMP, bone morphogenetic protein.
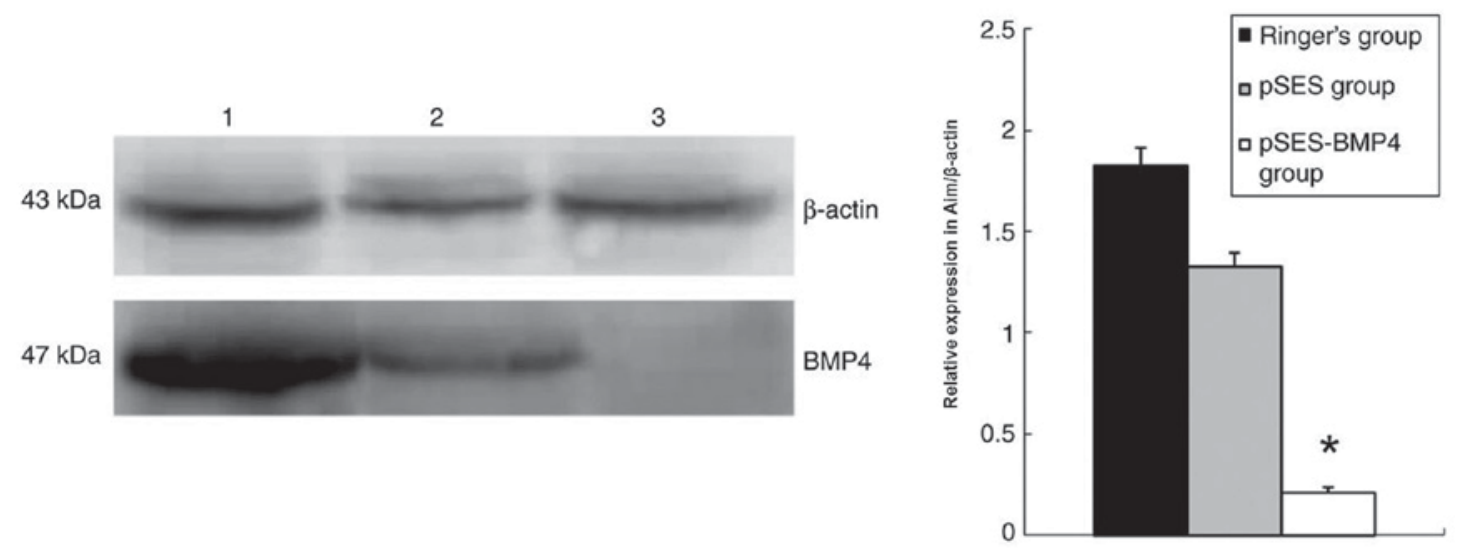

Figure 2. Western blot analysis of different groups of colon BMP4. The electropherogram derived from the western blot analysis of neurodevelopmental deficits associated protein BMP4 in the colons of three mouse groups. Lane 1: Ringer's group; Lane 2: pSES group; Lane 3: RNAi-BMP4 group. At the level of protein, RNAi-BMP4-induced mice, in contrast to the control group, significantly downregulated the levels of BMP4 in the colon. "P<0.05. BMP, bone morphogenetic protein; RNAi, RNA interference.

Statistical analysis. The RT-PCR and western blotting greyscale values were expressed as the mean \pm standard deviation. The differences among groups were analysed by a one-way analysis of variance and t-tests implemented in SPSS software version 17.0 (SPSS, Inc., Chicago, IL, USA), followed by Student-Newman-Keuls-q test. $\mathrm{P}<0.05$ was considered to indicate a statistically significant difference.

\section{Results}

BMP4 and downstream gene downregulation in F1 mouse colon is induced by administration of the siBMP4 plasmid to pregnant mice. PCR revealed that BMP4 and Smad4 miRNA in the colon was significantly lower in the RNAi-BMP4 group compared with the control and pSES groups at 7 days after birth ( $\mathrm{P}<0.05$; Fig. 1). A decrease in BMP4-Smad4 in mice may lead to aganglionosis (9-11). Western blotting results revealed that colon BMP4 in the RNAi-BMP4 group was significantly lower compared with the control and pSES groups (Fig. 2).

Formation of aganglionosis in F1 mice is induced by transplacental administration of RNAi-BMP4 to pregnant mice at 11.5 days. Barriers to stool discharge and abdominal distention were observed in BMP4 knockdown old mice $(\mathrm{n}=35 ; 10$ mice were sacrificed at 1 week). With a barium enema, giant colons were identified in 2-week-old mice from the RNAi-BMP4 group $(\mathrm{n}=23 ; 12$ mice were sacrificed at 1 week). Then, 4-week-old mice died from cerebral ischaemia and were autopsied ( $n=7$, some mice succumbed naturally). Unfortunately, the mouse rectum was so small that there was no suitable pressure probe. The spastic colon, transitional colonand giant colon were exposed (Fig. 3). With immunocytochemistry, a small number of glial-like cells were positive for $B M P 4$ in the colon of BMP4 knockdown mice. Additionally, absence or dysplasia of neurons was observed in RNAi-BMP4 colons.

\section{Discussion}

There area number of ways to establish aganglionosis in animal models (17-19). The principle methodis to affect the migration, differentiation and proliferation of NCSCs or artificially destroy the intestinal nervous system with drugs (20). A high folic acid (FA) diet during pregnancy leads to a gradual increase in serum FA in pregnant mice and their offspring, causing aganglionosis in the offspring (21). The level of FA in the offspring reached the highest value with $160 \mathrm{mg} / \mathrm{kg}$ FA feeding. It has also been demonstrated that a $0.1 \%$ benzalkonium 

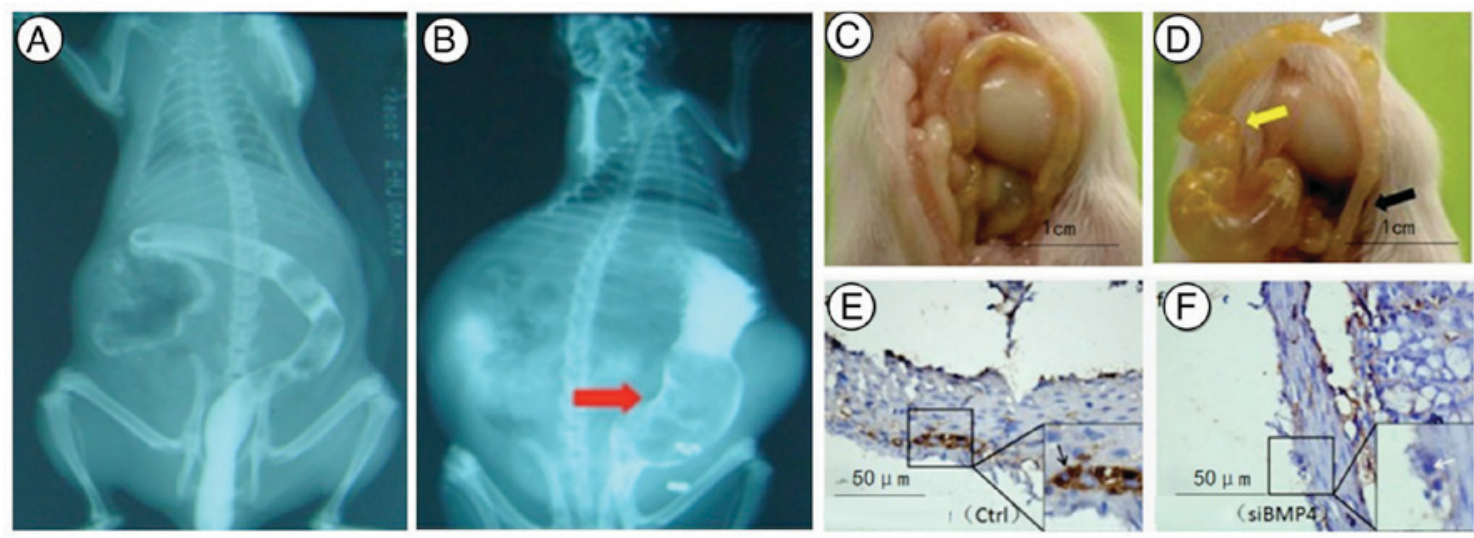

Figure 3. Colorectal shape was observed in the RNAi-BMP4 group, and immunohistochemistry staining of the colorectal region was performed in normal and RNAi-BMP4 tissues. (A) The barium enema form of a 3-week-old-mouse in the control group. (B) The barium enema form of a 3-week-old mouse in the RNAi-BMP4 group; the red arrow indicates the giant colon. (C) The mouse colon of the control group. (D) The mouse colon of the RNAi-BMP4 group. The black arrow indicates the spastic colon, the white arrow indicates the transitional colon, and the yellow arrow indicates the exposed giant colon. (E) In the colon of the control group, the expression of BMP4 was assayed by immunohistochemical staining. The Figure shows the normal neurons and nerve fibres. (F) The colon of the RNAi-BMP4 group, showing agenesis and absence of nerve fibres of neurons (magnification, x200). RNAi, RNA interference; BMP, bone morphogenetic protein.

chloride enema may also be used to establish aganglionos is in animal models. Ethylnitrosourea (ENU) is a type of artificial synthesised compound that leads to random and single-base mutations in a variety of organisms $(22,23)$. The offspring may end up with a severe mega colon phenotype due to ENU-induced mutations in C57BL/6 male mice. Trisomy 16 mice, which are likely to exhibit aganglionosis, are a type of genetic mouse model with clinical manifestations similar to those identified in the human trisomy 21 syndrome (24). This genetic mouse model, which does not express the endothelin 3 gene, is also known as piebald and spotted death mice and may develop defective intestinal aganglionic syndrome or congenital megacolon $(25,26)$. However, at present, there is a lack of a genetic mouse models that exhibits HD gene knockdown with unmistakeable implementation.

NCSCs originate from cells of the neural crest that migrate in chains as they colonize the embryonic gut, eventually forming the myenteric and submucosal plexus (27-29). Failure of the neural crest cells to colonize the gut leads to aganglionosis in the sigmoid colon, a pathological condition called Hirschsprung's disease, also known as congenital megacolon, in humans $(28,29)$. At present, the mechanism associated withthe signalling pathways that adjust NCSCs for migration to the intestinal tract and differentiation in the enteric nervous system remains unclear. The $B M P$ signalling pathway mayinvolve the migration process of NCSCs to the intestinal tract, in addition to the proliferation and differentiation of intestinal ganglion cells (30-32). Studies have suggested that BMP signalling serves an important role in differentiating NCSCs into enteric ganglia (8-12). Therefore, an improved understanding of the signalling pathways regulated by NCSCs and associated with the mechanisms of action is important to investigate the pathogenesis of aganglionosis. BMPs contribute to the largest subgroup of the TGF- $\beta$ super family and were originally identified by their ability to induce bone development $(27,28,31)$. Additionally, BMPs are expressed in the nervous system through out its differentiation. The mechanisms by which these BMPs regulate the induction of the neuroectoderm, the CNS primordium, and finally the neural crest, which gives rise to the NCSCs, have been reviewed $(11,33)$. Following neural tube closure, the most dorsal aspect of the tube becomes a signalling centre for BMPs, which directs the pattern of development of the dorsal spinal cord. Additionally, certain data suggested that BMP4 was a peripherally derived factor that may regulate the survival of motor neurons (34).

The RNAi phenomenon was identified in fungi and plants $(15,35)$. The placenta is responsible for transport between the mother and foetus and is a tissue barrier of high permeability $(14,16)$. The present study confirmed that plasmid vector injected into the tail vein of pregnant mice was able to be transferred to foetal mice through the blood-embryo barrier. The plasmid vector transfection of tissues and organs depends on the plasmid concentration, solvent volume, injection velocity, and weight of pregnant mice. The plasmid vector achieved good results when the injection concentrationswere $50 \mathrm{ng} / \mu 1$ and $10 \mu \mathrm{l} / \mathrm{g}$, and when the injection time was $5 \mathrm{sec}$. The majority of human aganglionosis cases have revealed an association with decreased BMP-Smad4 $(8,30,36)$. F1 mice that received transplacental RNAi-BMP4 at 11.5 days revealed disordered NCSCs. That is, the downregulation of BMP4 in the middle embryo stage possibly resulted in developmental problems in the peripheral nervous system. BMP4 was also involved in the TGF- $\beta / B M P / S m a d$-mediated signalling cascade as a transcriptional repressor of Smad proteins.

In summary, knockdown by transplacental RNAi is a powerful technique to study the effect of signalling pathways on responding tissues at the middle embryonic stage $(14,37)$. However, different genes regulate embryonic development through specific mechanisms, and different gene plasmids possess different transfection efficiencies. Thus, deciding the dose and when to intervene should be considered when exploring the function of a novel gene (38). The results presented here suggested that downregulation of the BMP4 transgene was an excellent prognostic factor of neurodevelopmental inactivity in mice. This approach may be used to make an aganglionosis 
mouse model. As the mouse colon is relatively short, it is planned to use rabbits to research colon gene expression in different regions in future studies.

\section{Acknowledgements}

This research was supported by National Natural Science Foundation of China (grant nos. 81370474 and 81600398).

\section{References}

1. Nakamura H, Henderson D and Puri P: A meta-analysis of clinical outcome of intestinal transplantation in patients with total intestinal aganglionosis. Pediatric Surg Int 33: 837-841, 2017.

2. Li MH, Eberhard M, Mudd P, Javia L, Zimmerman R, Khalek N and Zackai EH: Total colonic aganglionosis and imperforate anus in a severely affected infant with Pallister-Hall syndrome. Am J Med Genet A 167: 617-620, 2015.

3. Saida H, Hayet Z, Jamila C, Sana M, Samia B, Amine K, Badii H, Imed K, Lassad S, Mongi M et al: Familial near-total intestinal aganglionosis. J Neonatal Surg 6: 62-63, 2017.

4. Stenström P, Brautigam M, Borg H, Graneli C, Lilja HE and Wester T: Patient-reported Swedish nationwide outcomes of children and adolescents with total colonic aganglionosis. J Pediatr Surg 52: 1302-1307, 2017

5. Narayanan SK, Soundappan SS, Kwan E, Cohen RC, Charlton A and Cass DT: Aganglionosis with the absence of hypertrophied nerve fibres predicts disease proximal to rectosigmoid colon. Pediatr Surg Int 32: 221-226, 2016.

6. Teerlink CC, Bernhisel R, Cannon-Albright LA and Rollins MD A population-based description of familial clustering of Hirschsprung's disease. J Pediatr Surg S0022-3468: 30520-30521, 2017.

7. Cheng S, Wang J, Pan W, Yan W, Shi J, Guan W, Wang Y and Cai W: Pathologically assessed grade of Hirschsprung-associated enterocolitis in resected colon in children with Hirschsprung's disease predicts postoperative bowel function. J Pediatr Surg 52: 1776-1781, 2017.

8. Chiou J, Su CY, Jan YH, Yang CJ, Huang MS, Yu YL and Hsiao M: Decrease of FSTL1-BMP4-Smad signaling predicts poor prognosis in lung adenocarcinoma but not in squamous cell carcinoma. Sci Rep 7: 9830, 2017.

9. Modica S and Wolfrum C: The dual role of BMP4 in adipogenesis and metabolism. Adipocyte 6: 141-146, 2017.

10. Nemashkalo A, Ruzo A, Heemskerk I and Warmflash A: Morphogen and community effects determine cell fates in response to BMP4 signaling in human embryonic stem cells. Development 144: 3042-3053, 2017.

11. Sprott D and Chavakis T: A BMP4-angiomiR connection in angiogenesis. Thromb Haemost 117: 650-650, 2017.

12. Salie R, Niederkofler V and Arber S: Patterning molecules; multitasking in the nervous system. Neuron 45: 189-192, 2005.

13. Soares ML, Haraguchi S, Torres-Padilla ME, Kalmar T, Carpenter L, Bell G, Morrison A, Ring CJ, Clarke NJ, Glover DM and Zernicka-Goetz M: Functional studies of signaling pathways in peri-implantation development of the mouse embryo by RNAi. BMC Dev Biol 5: 28, 2005.

14. Jin X, Chen Z, Xiang L, Luo Q, Guo Z, Ding X and Jin X: Colorectal polyp model established by transplacental BMP4 RNAi. Mol Med Rep 10: 33-38, 2014.

15. O'shea KS, De Boer LS, Slawny NA and Gratsch TE: Transplacental RNAi: Deciphering gene function in the postimplantation-staged embryo. J Biomed Biotechnol 2006: 18657, 2006.

16. Gratsch TE, De Boer LS and O'Shea KS: RNA inhibition of BMP-4 gene expression in postimplantation mouse embryos. Genesis 37: 12-17, 2003.

17. Heanue TA, Boesmans W, Bell DM, Kawakami K, Vanden Berghe P and Pachnis V: A novel Zebrafish ret heterozygous model of Hirschsprung disease identifies a functional role for mapk10 as a modifier of enteric nervous system phenotype severity. PLoS Genet 12: e1006439, 2016.

18. Gariepy CE, Williams SC, Richardson JA, Hammer RE and Yanagisawa M: Transgenic expression of the endothelin-B receptor prevents congenital intestinal aganglionosis in a rat model of Hirschsprung disease. J Clin Invest 102: 1092-11101, 1998.
19. Gasc JM, Clemessy M, Corvol P and Kempf H: A chicken model of pharmacologically-induced Hirschsprung disease reveals an unexpected role of glucocorticoids in enteric aganglionosis. Biol Open 4: 666-671, 2015.

20. Villalba-Benito L, Torroglosa A, Fernández RM, Ruíz-Ferrer M, Moya-Jiménez MJ, Antiñolo G and Borrego S: Overexpression of DNMT3b target genes during enteric nervous system development contribute to the onset of Hirschsprung disease. Sci Rep 7: $6221,2017$.

21. Wu CQ, et al: Mice models of congenital magacolon induced by high-dose folic acid diet at the duration of pregnancy. Chinese Journal of Experimental Surgery 28: 1925-1926, 2011.

22. Wagner JP, Sullins VF and Dunn JC: A novel in vivo model of permanent intestinal aganglionosis. J Surg Res 192: 27-33, 2014

23. Fujimura T, Shibata S, Shimojima N, Morikawa Y, Okano H and Kuroda T: Fluorescence visualization of the enteric nervous network in a chemically induced aganglionosis model. PLoS One 11: e0150579, 2016

24. Li J, Busch LC and Kunel W: The development of enteric nervous system in terisomy 16 mice with the occurrence of congenital megacolon. National Med J China 79: 466-469, 1999.

25. Chen B, Ouyang HL, Wang WH, Yin YH, Yan LN, Yang B and Xue ZF: Hirschsprung disease is associated with an L286P mutation in the fifth transmembrane domain of the endothelin-B receptor in the N-ethyl-N-nitrosourea-induced mutant line. Exp Anim 65: 245-251, 2016.

26. Frykman PK, Cheng Z, Wang X and Dhall D: Enterocolitis causes profound lymphoid depletion in endothelin receptor Band endothelin 3-null mouse models of Hirschsprung-associated enterocolitis. Eur J Immunol 45: 807-817, 2015.

27. Rollo BN, Zhang D, Stamp LA, Menheniott TR, Stathopoulos L, Denham M, Dottori M, King SK, Hutson JM and Newgreen DF: Enteric neural cells from Hirschsprung disease patients form Ganglia in autologous aneuronal colon. Cell Mol Gastroenterol Hepatol 2: 92-109, 2016

28. Wang Y, He T, Liu J, Liu H, Zhou L, Hao W, Sun Y and Wang X: Synergistic effects of overexpression of BMP2 and TGF $\beta 3$ on osteogenic differentiation of bone marrow mesenchymal stem cells. Mol Med Rep 14: 5514-5520, 2016.

29. Li Y, Yao D, Zhang J, Liu B, Zhang L, Feng H and Li B: The effects of epidermal neural crest stem cells on local inflammation microenvironment in the defected sciatic nerve of rats. Front Mol Neurosci 10: 133, 2017.

30. Goldstein AM, Brewer KC, Doyle AM, Nagy N and Roberts DJ: BMP signaling is necessary for neural crest cell migration and ganglion formation in the enteric nervous system. Mech Dev 122: 821-833, 2005.

31. KuroyanagiG,TokudaH, Yamamoto N,Matsushima-NishiwakiR, Mizutani J, Kozawa O and Otsuka T: Resveratrol amplifies BMP-4-stimulated osteoprotegerin synthesis via p38 MAP kinase in osteoblasts. Mol Med Rep 12: 3849-3854, 2015.

32. Pavelock KA, Girard BM, Schutz KC, Braas KM and May V: Bone morphogenetic protein down-regulation of neuronal pituitary adenylate cyclase-activating polypeptide and reciprocal effects on vasoactive intestinal peptide expression. J Neurochem 100: 603-616, 2007.

33. Weber M, Apostolova G, Widera D, Mittelbronn M, Dechant G, Kaltschmidt B and Rohrer H: Alternative generation of CNS neural stem cells and PNS derivatives from neural crest-derived peripheral stem cells. Stem Cells 33: 574-588, 2015.

34. Chou HJ, Lai DM, Huang CW, McLennan IS, Wang HD and Wang PY: BMP4 is a peripherally-derived factor for motor neurons and attenuates glutamate-induced excitotoxicity in vitro. PLoS One 8: e58441, 2013.

35. Torres-Martinez S and Ruiz-Vazquez RM: The RNAi Universe in Fungi: A varied landscape of small RNAs and biological functions. Annu Rev Microbial 71: 371-391, 2017.

36. Hegarty SV, O'Keeffe GW and Sullivan AM: BMP-Smad 1/5/8 signalling in the development of the nervous system. Prog Neurobiol 109: 28-41, 2013

37. Zou GM, Yu J, LeBron C and Fu Y: RNAi knockdown of Apel gene in the differentiation of mouse embryonic stem cells. Methods Mol Biol 1622: 131-138, 2017.

38. Jin, $\mathrm{X}$, et al: Down-regulation of the target genes using trans-placental RNAi. J Med Mol Bio 14: 6-9, 2017. 